Itinéraires Itinéraires

Littérature, textes, cultures

\title{
Les écrivains communistes pour la jeunesse pendant l'entre-deux-guerres
}

\author{
Mathilde Lévêque
}

\section{(2) OpenEdition}

\section{Journals}

Édition électronique

URL : http://journals.openedition.org/itineraires/1400

DOI : $10.4000 /$ itineraires. 1400

ISSN : 2427-920X

Éditeur

Pléiade

\section{Édition imprimée}

Date de publication : 1 décembre 2011

Pagination : 105-115

ISBN : 978-2-296-55744-4

ISSN : 2100-1340

\section{Référence électronique}

Mathilde Lévêque, "Les écrivains communistes pour la jeunesse pendant l'entre-deux-guerres », Itinéraires [En ligne], 2011-4 | 2011, mis en ligne le 01 décembre 2011, consulté le 30 avril 2019. URL: http://journals.openedition.org/itineraires/1400 ; DOI : 10.4000/itineraires.1400

\section{(C) $(\oplus \Theta$}

Itinéraires est mis à disposition selon les termes de la licence Creative Commons Attribution - Pas d'Utilisation Commerciale - Pas de Modification 4.0 International. 


\title{
Les écrivains communistes pour la jeunesse pendant l'entre-deux-guerres
}

\begin{abstract}
After the First World War, communist writers participate in a novel impetus towards the reflexion about and the creation of children literature. As engaged militants and fellow travelers, these writers create new heroes that fight against capitalism and work for peace or for the liberation of colonised people. In their innovative books, translation and cultural exchange play moreover an important dynamical role.
\end{abstract}

Keywords : children's literature, youth, pacifism, anticolonialism, Vaillant-Couturier Mots clés : littérature de jeunesse, enfance, pacifisme, anticolonialisme, VaillantCouturier

Période encore mal connue de l'histoire de la littérature pour la jeunesse, l'entre-deux-guerres, loin d'être uniquement marquée par le marasme économique et le déclin esthétique, est une étape importante dans la réflexion autour de la littérature pour la jeunesse, dans la création de nouveaux livres pour enfants, dans l'amorce d'un renouveau de l'écriture narrative et romanesque. Le communisme, qui se développe et s'affirme en France au lendemain de la Première Guerre mondiale, participe à ce nouvel élan de réflexion et de création, croisant l'engagement politique et l'écriture, l'intérêt pour l'enfance et la jeunesse et le discours de propagande. Dès 1921, Paul Vaillant-Couturier, en publiant le récit pacifiste Jean-sans-Pain ${ }^{1}$, montre l'intérêt que représente la littérature de jeunesse pour les écrivains communistes, en mêlant la recherche d'une écriture nouvelle à la nécessité de proposer un discours politique en direction des jeunes lecteurs. Qui sont, au cours des années 1920 et 1930, les écrivains communistes qui ont choisi l'écriture pour la jeunesse? Quelles sont leurs œuvres et comment ont-elles été diffusées? Comment s'articulent les préoccupations idéologiques et

1. Paul Vaillant-Couturier, Jean-sans-Pain: Histoire pour tous les enfants, racontée par Paul Vaillant Couturier devant les images de Picart le Doux, Paris, Clarté, 1921. 
la modernité d'une écriture dont l'enjeu politique semble de plus en plus pressant au long des années 1930 ?

Membres du Parti communiste, du simple militant au cadre, plusieurs écrivains comprennent très tôt la nécessité de s'adresser aux enfants, non seulement par le biais du documentaire ou du témoignage, mais aussi, et surtout, par celui de la fiction. Paul Vaillant-Couturier apparait comme le premier écrivain communiste à avoir saisi l'urgence d'une parole destinée aux plus jeunes : avec Jean-sans-Pain, paru en 1921 aux Éditions Clarté il propose une histoire en images, illustrée par Picart Le Doux, en grand format $(24 \times 31 \mathrm{~cm})$. Reprenant le motif de l'orphelin, fréquemment utilisé en littérature pour la jeunesse, Paul Vaillant-Couturier crée un récit original où le merveilleux côtoie le réalisme : guidé par un lièvre magique dont les ailes se dévissent pour former l'hélice d'un aéroplane, Jean, dont le père a été tué dans les tranchées et la mère est morte d'épuisement et de misère, découvre le monde inhumain et dangereux de l'usine, la richesse révoltante des nantis au soir de Noël, l'horreur de la guerre et sa folie meurtrière, avant de s'envoler vers l'Est, vers un pays de rêves et d'espoir. Réédité en 1933 aux Éditions sociales internationales, le récit, désormais illustré par Jean Lurçat, est prolongé par un épisode révolutionnaire faisant plus explicitement référence à la révolution bolchevique : Jean-sans-Pain et ses camarades ouvriers et soldats prennent les armes pour chasser les riches et lâches bourgeois qui les exploitaient. En novembre 1937, le journal Mon Camarade, « illustré de la jeunesse », rend hommage à Paul Vaillant-Couturier, ce « grand ami » des enfants, écrivain et bienfaiteur, mort brusquement au mois d'octobre précédent :

Paul Vaillant-Couturier avait été en effet le fondateur de magnifiques œuvres pour l'enfance, dans sa municipalité de Villejuif, et il avait veillé personnellement à l'organisation d'une chorale enfantine modèle. Vaillant, dans sa vie active et multiple avait trouvé le temps d'écrire des livres pour l'enfance : Anne pauvre et cochon gras (dans les Livrets de Mon Camarade) et Jean-sans-Pain. Ces deux livres sont de vrais chefs-d'œuvre, et sont aimés par tous les enfants ${ }^{3}$.

$\mathrm{Si}$ « tous les enfants » mentionnés par le rédacteur de Mon Camarade ne sauraient rassembler tous les jeunes lecteurs français, si les louanges confinent au panégyrique, voire au discours de propagande, il reste que

2. «À la suite du mouvement intellectuel Clarté, créé en mai 1919, et de sa revue, fondée en octobre, le mouvement se dote d'une maison d'édition, «Éditions Clarté », à la fin de l'année 1921. Elle répond aux ambitions éducatives du mouvement. C'est une production moins importante que la Librairie du Travail et la Bibliothèque communiste (six titres en 1921) et surtout plus littéraire, marquée par le pacifisme » (Marie-Cécile Bouju, « Le livre comme arme de propagande : le cas des relations entre le service d'édition de l'Internationale communiste et la France (1919-1939) », Communisme, no 97-98, 2009, p. 9).

3. Mon Camarade, l'illustré de la jeunesse, 18 novembre 1937. 
Paul Vaillant-Couturier est l'un des rares écrivains et militants communistes à avoir non seulement choisi d'écrire pour les enfants, mais aussi à avoir réfléchi sur une écriture plus moderne. Loin des poncifs hérités du XIX ${ }^{\mathrm{e}}$ siècle, l'écrivain adopte une langue qui se refuse aux propos bêtifiants, pour donner à lire une œuvre qui montre les prémices d'une réflexion sur les relations entre le texte et l'image, et qui contribue, au moins en partie, à l'avènement, quelques années plus tard, de l'album moderne pour enfants. Les images de Picart Le Doux ne sont pas des simples illustrations du texte de Paul Vaillant-Couturier : l'iconographie s'intègre dans le corps du texte, l'encadre, le met en scène. Dans la scène de rencontre entre le lièvre et Jean, le texte, placé à droite du lièvre qui, de dos, parle à Jean, fonctionne presque comme un phylactère de l'image : " C'est toi qui dois apprendre ce soir la vérité à ceux d'entre nous qui souffrent le plus. Et alors, toi, Jean-sans-Pain, pour la première fois de ta petite vie, tu souri$\operatorname{ras}^{4}{ }^{\prime}$. Quelques pages plus loin, la transformation du lièvre et des perdrix en aéroplane fonctionne de façon parallèle dans le texte et dans l'image : « Les perdreaux battent des ailes... Alors Jean se sent léger, léger. Le vent souffle plus fort... Jean monte sur la route des héros, des alouettes et des canards ». L'image s'élève également sur l'espace de la page, tandis que l'envol progressif des oiseaux semble reprendre, graphiquement, les phrases courtes, les répétitions et les points de suspension. L'album et son esthétique ne sont pas loin : Paul Vaillant-Couturier et Picart Le Doux ont compris que l'interaction du texte et de l'image, si elle prend du sens sur l'espace de la page, peut en avoir également d'une page à l'autre, dans le simple fait de la tourner. Après l'envol magique, le lecteur tourne la page, pour voir planer, sur la partie supérieure, l'aéroplane. Magie du livre d'images, magie de l'album : en tournant la page, le lecteur a suivi et opéré la transformation des animaux en avion, il est pleinement entré dans le monde du rêve et de l'imaginaire. En 1931, une dizaine d'années plus tard, Jean de Brunhoff comprendra également qu'en tournant une page, un petit éléphant né dans la grande forêt grandit sous les yeux de l'enfant ${ }^{5}$. Jean-sans-Pain, l'un des premiers livres pour enfants à dénoncer la folie meurtrière de la guerre, montre enfin une écriture moderne, où l'héritage du conte merveilleux et les traces d'oralité, loin d'être des archaïsmes, dessinent l'esquisse d'une réflexion nouvelle sur l'art de conter et de raconter. " C'est très laid la guerre » : quand Jean arrive près des tranchées, Paul Vaillant-Couturier choisit moins de décrire que de faire entendre des sons discordants : « Fuuuui baoum! Boum! Boum! Boum! Tatatatatatatac! Ziiiiiiiii clac! Rrrrraou! Rrrrraou! La terre sautait. On était à la guerre maintenant, et en

4. Paul Vaillant-Couturier, op. cit., p. 11.

5. Jean de Brunhoff, Histoire de Babar, le petit éléphant, Paris, Le Jardin des modes, 1931. Je renvoie aux analyses d'Isabelle Nières-Chevrel publiées dans plusieurs articles, dont " Jean de Brunhoff : inventer Babar, inventer l'espace », La Revue des livres pour enfants, no 191, février 2000, p. 109-120. 
plein, tu sais ${ }^{6} »$. L'adresse au lecteur, marque d'oralité héritée de la prise de parole du conteur, entraîne plus directement au centre de la scène, conduisant le jeune lecteur dans un univers de bruits et de violence où fil de fer barbelé, glace, gros obus et baïonnette prennent la parole en blessant cruellement Jean à chaque pas. Jean, « les mains écorchées, les pieds trempés et déchirés, le tablier en lambeaux, le pantalon jaune tout percé », suit son chemin de croix avant d'opérer sa transfiguration finale : au soir de Noël, il réconcilie « Boches » et « Franzouses », pauvres soldats des tranchées, qui s'agenouillent religieusement devant Jean, « l'Enfant qui doit venir $»^{7}$. Le récit, qui fait aussi explicitement référence à la naissance de Jésus, présenté lui aussi comme un pauvre messager de paix, comporte une dimension christique très évidente, qui n'est pas incompatible avec le propos communiste. Ce que Paul Vaillant-Couturier dénonce, ce sont les hypocrisies de la religion, la richesse du clergé et ses mensonges, et non le message de paix et de fraternité du Christ.

Le propos politique demeure clair et évident dans Jean-sans-Pain: le pacifisme se conjugue avec une dénonciation du capitalisme, qui prend la forme d'une lutte armée dans la réécriture de 1933, rendant le propos pacifiste plus problématique. Le combat contre le capitalisme permet aux écrivains communistes pour la jeunesse d'opposer de façon manichéenne bons et méchants, comme dans Le Mystère du serpent à plumes (1935), premier épisode des aventures de Pierrot Lancry, écrit par Georges Sadoul sous le pseudonyme de René Duchâteau ${ }^{8}$. Reprenant le schéma du roman populaire, cette histoire pour adolescents est publiée tout d'abord sous la forme d'un feuilleton entre octobre 1935 et octobre 1936 dans Mon Camarade. Pierrot Lancry, 13 ans, le regard noir et franc, est le fils d'un ouvrier travaillant dans une raffinerie, près de Rouen. Son oncle, physicien et savant, est soudain enlevé par les sbires du puissant et richissime roi du pétrole, Vandergold, qui contrôle toute une partie de la presse, complice de ses machinations criminelles : seuls les journaux de gauche sont désignés comme des journaux indépendants. Dans un rythme rapide et trépidant, le roman voit entrer en scène les nazis, poursuivant le Professeur Schulz, physicien hambourgeois en exil, puis les Russes blancs, alliés des « hitlériens » décidés à détruire le régime soviétique, les riches et cruels bourgeois en villégiature à Penthièvre, qui n'hésitent pas à tirer comme des lapins des enfants échappés d'une maison de correction, «bagne d'enfants ». Le récit se poursuit au rythme d'un rebondissement par chapitre, selon la structure du roman-feuilleton, qui sait ménager le suspense à la fin de chaque épisode. La lutte contre l'exploitation et la persécution des ouvriers, mais aussi des hommes de science par le capitalisme et le nazisme, prend ici la

6. Paul Vaillant-Couturier, op. cit., p. 35.

7. Ibid., respectivement p. 37 et p. 45.

8. René Duchâteau, Le Mystère du serpent à plumes : Grand roman d'aventures. Avec des illustrations de Janser, Paris, Éditions sociales internationales, 1935. 
forme d'un roman à suspense, fait d'enlèvements et de courses-poursuites, où l'esthétique du roman populaire et du roman d'aventures, en pleine expansion pendant l'entre-deux-guerres ${ }^{9}$, semble venir rencontrer celle, plus récente, du cinéma.

Le colonialisme, qui a nourri bon nombre de romans d'aventures, apparaît comme cette autre forme d'exploitation combattue par les écrivains communistes. Encore rares dans l'entre-deux-guerres, les récits anticolonialistes pour la jeunesse apparaissent sous la plume d'écrivains communistes, comme René Maublanc (1891-1960), philosophe engagé dans le mouvement anticolonial et le mouvement antifasciste, et qui fait partie, selon Marie-Cécile Bouju, des dix auteurs communistes les plus publiés en France entre 1935 et $1938^{10}$. Derradji, publié chez Larousse en 1927, raconte le séjour en France d'un jeune Algérien de 13 ans, Derradji ben Saoudi, fils d'un « riche indigène », le plus gros propriétaire de palmeraies d'El Oued, au Sahara. Ami de Jacques, fils de l'instituteur M. Lehuédé, Derradji traverse la Méditerranée, découvre Marseille puis la Bretagne de Bourg-de-Batz, près du Croisic. Le roman opère plusieurs rapprochements tendant à montrer une certaine égalité entre Français et Arabes : Derradji et Francis, cousin de Jacques, remarquent ainsi les similitudes entre la vie dans le désert et la vie en mer. Pourtant, René Maublanc montre également aux jeunes lecteurs les limites de ce rapprochement et l'hostilité toujours sousjacente entre Français et Algériens : un jour où il se met en colère contre Derradji qui maltraite un crabe, $M$. Lehuédé se laisse emporter et s'écrie :

\footnotetext{
— Tu t'amuses à faire le mal, à tourmenter ceux qui sont plus faibles que toi. Tu es brutal. C'est naturel, après tout; tu n'es pas Arabe pour rien.

Du coup, Derradji bondit. D'une voix rauque, il cria :

- Si les Arabes sont brutaux, c'est que les « Roumis » le leur ont appris. Tu m'attrapes ici pour avoir fait du mal à une bête. Mais, chez nous, est-ce que tu attrapes les Français qui font du mal aux Arabes ${ }^{11}$ ?
}

La scène se termine brutalement par une paire de gifles, la violence l'emportant sur la parole et la discussion. Devant sa nièce Yvonne, amie de Derradji, M. Lehuédé reconnaît ses torts :

\footnotetext{
- Ce qui m'a exaspéré tout à l'heure, c'est justement que Derradji disait des choses trop vraies. Nous donnons trop souvent aux Arabes, en Algérie, l'exemple de la brutalité et de la violence. Nous les traitons comme une race inférieure, et nous ne nous apercevons pas que c'est par notre faute qu'ils
}

9. Voir Matthieu Letourneux, Le Roman d'aventures, Limoges, Pulim, coll. « Médiatextes », 2010.

10. Marie-Cécile Bouju, La Production des maisons d'éditions du P.C.F., 1921-1956, Mémoire d'étude pour l'obtention du diplôme de conservateur de bibliothèques, sous la direction de Jean-Yves Mollier, Université de Versailles-Saint-Quentin-en-Yvelines, 1999, p. 44.

11. René Maublanc, Derradji, Paris, Larousse, 1927, p. 133. 
restent, à certains égards, inférieurs à nous. Je sais bien que nous sommes quelques-uns à vouloir réagir, à essayer de les conquérir vraiment, par le cœur et non plus seulement par la force ou par l'intérêt. Mais ce qui me désole, c'est qu'au moindre conflit, la vieille haine reparaît. Tout à l'heure, sur la plage, il n'y avait plus Lehuédé et Derradji, le maître et l'élève, les deux amis. Il y avait un sujet dressé contre son vainqueur, le «Bicot» dressé contre le « Roumi ».

- Mais cela va changer, n'est-ce pas, tonton René?

- Je l'espère, mon enfant. Mais il faudra du temps, et tellement d'efforts d'un côté comme de l'autre! Il faudra une fusion plus complète des deux civilisations. Il faudra plus d'écoles. Des voyages, comme celui de Derradji. Et aussi des mariages entre les meilleurs des petits Arabes et nos jeunes filles de France.

M. Lehuédé ne s'aperçut pas qu'Yvonne avait rougi ${ }^{12}$.

Cas extrêmement rare dans la littérature pour la jeunesse, le roman de René Maublanc ne se contente pas de prôner le rapprochement entre les Français et les Algériens; il milite en faveur d'une assimilation entre les deux peuples et les deux civilisations, allant jusqu'à proposer des mariages mixtes entre Algériens et Françaises. L'amitié très forte qui unit Derradji et Yvonne semble préfigurer fortement cet avenir que seul un roman peut imaginer. Alors que l'entre-deux-guerres est marquée par une expansion du roman colonial, pour la jeunesse comme pour les adultes, un écrivain communiste décide d'écrire un roman pour la jeunesse allant à contrecourant des clichés et des idées dominantes.

Les écrivains qu'il est possible de classer sous l'appellation d' " écrivains communistes » ne sont pas tous militants ou membres du Parti : en littérature pour la jeunesse comme en littérature générale, il faut compter avec les compagnons de route, séduits par un discours humaniste plus que par des doctrines, $\mathrm{y}$ trouvant une foi dans l'enfance et dans l'avenir qu'ils cherchent à traduire dans leurs ouvrages. Charles Vildrac (1882-1971) est l'une de ces figures de la littérature pour la jeunesse : fils d'un ancien communard et d'une directrice d'école, utopiste et poète, Vildrac crée avec son roman L'Île Rose (1924), illustré par Edy-Legrand, l'une des premières utopies littéraires pour la jeunesse. Loin de la misère et de la grisaille des grandes villes, un riche philanthrope, l'« Enchanteur », crée sur une île au large de la Méditerranée une école idéale, où les petits garçons vivent et grandissent en harmonie avec une nature généreuse, dans le partage, l'entraide et la solidarité. Île rose mais non île rouge, l'utopie de Vildrac est néanmoins reprise par Georges Sadoul qui en publie de larges extraits dans Mon Camarade : le discours de propagande a tôt fait de créer le lien entre la fiction et l'image d'une réalité fabriquée par le discours officiel d'un parti soumis à Moscou. La présentation de ce texte dans la rubrique « Notre page choisie », en janvier 1939, alors que Vildrac s'affirme désormais comme un 
compagnon de route du Parti communiste - ce qu'il n'était pas en 1924 -, opère un glissement facile du rose au rouge, et retour :

Hélas! Le temps des magiciens est-il passé? Que non! Charles Vildrac, qui vient de passer plusieurs mois en Russie, a pu voir que L'Île Rose de son rêve était devenu le continent rouge de l'enfance heureuse, et les 15.000 enfants de la banlieue rouge n'ont-ils pas vécu, au cours de ces dernières vacances, quelques semaines dans leur île Rose ${ }^{13}$ ?

Le discours de propagande trouve dans la fiction littéraire et en particulier dans l'utopie un support idéal : le rêve né de l'imagination d'un poète est désormais devenu réalité grâce à l'action du grand frère russe. Ce passage du rêve à la réalité est implicitement mis en relation avec le compagnonnage de l'écrivain : le voyage en Union soviétique de Vildrac, rapproché de la publication de ses textes dans Mon Camarade, fonctionne comme la figuration métaphorique d'un processus de transformation de la littérature en expérience vécue. De fait, Vildrac s'est rendu deux fois en Union soviétique, en 1929 et en 1935, et son récit de voyage Russie neuve ${ }^{14}$ témoigne de cette expérience. Le titre de cet ouvrage fait référence au «Cercle de la Russie neuve », créé en 1927 à l'initiative d'intellectuels membres ou proches du Parti communiste, et qui, « à travers des commissions scientifiques, littéraires ou artistiques, se proposait de propager le marxisme et les réalisations de l'URSS $^{15} \gg$. Charles Vildrac fait partie de ce cercle, aux côtés, entre autres, de Paul Langevin, Claude Autant-Lara, Henri Wallon, René Maublanc, Francis Jourdain. Du rose au rouge, l'utopie littéraire de Vildrac a dépassé la fiction et le merveilleux, grâce à la force du communisme russe, comme si le jeune lecteur pouvait désormais traverser l'espace fictionnel pour accéder à une réalité d'autant plus supérieure qu'elle est un réel bâti au-delà du rêve et des frontières mêmes de l'imagination. Une fiction qui n'avait à l'origine aucune finalité politique précise se trouve ainsi totalement réinvestie par le discours de propagande.

Autre compagnon de route, écrivain majeur de l'entre-deux-guerres, Romain Rolland s'est intéressé de près à la littérature pour la jeunesse. Si celle-ci n'est pas au centre de son œuvre d'écrivain, elle demeure une préoccupation périphérique importante : Romain Rolland cautionne ainsi une adaptation de Jean-Christophe en livre de lecture ${ }^{16}$, il possède lui-même plusieurs livres pour enfants, comme le montre l'examen attentif du fonds R-ROLLAND de la Bibliothèque nationale de France. Ce fonds, d'une très

13. Mon Camarade, janvier 1939, p. 5.

14. Charles Vildrac, Russie neuve, Paris, Éditions Émile-Paul frères, 1935.

15. Noël Terrot, Histoire de l'éducation des adultes en France, Paris, L'Harmattan, 1997, p. 198.

16. Jean-Christophe de Romain Rolland, présenté aux enfants par $\mathrm{M}^{\mathrm{me}}$ Hélier-Malaurie, livre de lecture, certificat d'études, classes de $6^{\text {e }}$, illustrations de Ray-Lambert, Paris, Albin Michel, 1932. 
grande richesse, présente une masse considérable d'imprimés, puisque plus de 13000 volumes y sont catalogués et cotés. Outre les œuvres de Romain Rolland, il se compose d'ouvrages faisant partie de la bibliothèque de l'écrivain : dans cet ensemble très important, quelques livres pour la jeunesse apparaissent, principalement des livres français et allemands écrits et publiés pendant l'entre-deux-guerres par des écrivains communistes ou proches du Parti communiste, comme ceux de Charles Vildrac, La Colonie (1930), Milot (1933), Bridinette (1935), de Paul Vaillant-Couturier (l'édition de 1933 de Jean-sans-Pain), de Léopold Chauveau, Les Deux font la paire (1937), de Claude Aveline, Baba Diène et Morceau-de-Sucre (1937), ou encore l'essai de Georges Sadoul, Ce que lisent vos enfants (1938). Parmi les romans pour la jeunesse allemands, il faut aussi noter Hans et son lièvre enchanté, traduction par Pierre Kaldor du récit de Lisa Tetzner, Hans Urian, inspiré de Jean-sans-Pain, et le roman d'Alex Wedding, Das Eismeer ruft (1936), dédicacé à Romain Rolland de la main même de l'auteur. Enfin, Romain Rolland écrit un texte pour enfants, texte historique et non texte de fiction, Valmy, illustré par Jean Trubert et publié aux Éditions sociales internationales en 1938. Dès les premières lignes, l'auteur trace un lien explicite entre l'histoire de la Révolution française et l'histoire contemporaine :

L'histoire n'est pas un recueil d'anecdotes et de récits romancés. Elle est une somme d'expériences humaines, dont la connaissance exacte éclaire non seulement le passé, mais le présent, et doit guider nos pas.

L'histoire de la France pendant la Révolution française a beaucoup de rapports avec celle de la France, de la Russie et de l'Espagne d'aujourd'hui ! Qu'elle nous instruise et nous inspire ${ }^{17}$ !

L'écriture pour la jeunesse de Romain Rolland est essentiellement politique et pédagogique : en 1938, elle semble dictée par l'urgence des événements, la montée des périls et la préparation, sous-jacente mais réelle, de la jeunesse à s'engager dans une nouvelle lutte, l'ouvrage se terminant sur cet appel : " Fils de la Révolution, vous d'aujourd'hui, êtesvous encore capables d'entendre, sans gêne et sans peur, ces fiers échos de la canonnade de Valmy ${ }^{18}$ ? $\gg$ Le pacifisme d'un Jean-sans-Pain semble loin désormais : en 1938, les appels à la paix font de nouveau place aux échos de canons qui, bientôt, ne seront plus seulement ceux des livres d'histoire et des livres d'images.

Enfin, pour compléter la présentation des écrivains communistes pour la jeunesse de l'entre-deux-guerres, il faudrait en mentionner plusieurs qui, sans être ni communistes ni officiellement compagnons de route du Parti, choisissent de publier leurs ouvrages pour enfants chez des éditeurs communistes comme les Éditions sociales internationales. Tel est le cas de

17. Romain Rolland, Valmy, illustrations de Jean Trubert, Paris, Éditions sociales internationales, 1938, p. 1.

18. Ibid., p. 30 . 
Léopold Chauveau, écrivain malheureusement méconnu de l'entre-deuxguerres, auteur de récits courts où l'imagination et l'humour mettent peu à peu en place une forme de fantasy à la française, sur le modèle de Lewis Carroll. Parfois perçu comme le "Kipling français », Léopold Chauveau manie avec autant de plaisir et de talent le conte étiologique et la caricature, le discours humaniste et l'humour, en s'adressant à l'enfant et en particulier aux plus jeunes comme à des égaux, leur laissant la parole comme dans Les Deux font la paire ${ }^{19}$. Autre exemple, Colette Vivier semble trouver dans l'édition communiste de nouvelles pistes narratives : quittant les thématiques du roman bourgeois pour enfants, elle cherche à créer des personnages plus proches du peuple et du monde ouvrier, qu'elle avoue ne pas connaître. Parallèlement, elle cherche de nouveaux modes d'écriture, plus libres, plus modernes. Elle s'essaie ainsi à la forme du journal fictif, en publiant des textes dans Mon Camarade. Le numéro du 12 août 1937 contient un texte qui n'est autre qu'une première version de La Maison des petits bonheurs ${ }^{20}$. Le texte est plus court que le roman, le récit est moins complexe, mais on trouve en germe tous les éléments qui ont fait le succès du roman des années 1939-1940, toujours recommandé par les listes officielles du ministère de l'Education nationale : sous la forme d'un journal intime, une petite fille, issue d'une modeste famille d'artisans parisiens, raconte avec ses mots d'enfant sa vie quotidienne, les anecdotes de son quartier, ses joies et ses peines d'écolière. Sorte d'essai avant la rédaction de La Maison des petits bonheurs, le « Journal de Riquette » est écrit par Marie-Jeanne, 11 ans, ainsi surnommée. Le choix d'un journal communiste s'expliquerait, peut-être, par la possibilité d'une certaine marge de liberté pour un écrivain qui tente de changer d'écriture, non seulement d'un point de vue thématique mais aussi d'un point de vue formel. La publication de textes de Colette Vivier dans Mon Camarade peut également s'inscrire dans la volonté de Georges Sadoul, créateur du journal, de proposer aux jeunes lecteurs des textes de littérature non seulement engagée, mais plus largement de littérature classique et contemporaine, en maintenant une certaine exigence de qualité esthétique.

Dans les années 1920 est lancée une première expérience de périodique communiste pour la jeunesse, Le Jeune Camarade, « journal international des enfants ouvriers ", qui était, selon Marie-Cécile Bouju, « en quelque sorte une édition à peine adaptée de L'Avant-garde pour les enfants ${ }^{21} \gg$. Suite à cette première expérience sur laquelle on connaît fort peu de choses, Mon Camarade est créé en 1933 par Georges Sadoul et Marcelle Hirsum et édité par la Fédération d'enfants ouvriers et paysans. Dès août 1933, le périodique

19. Léopold Chauveau, Les Deux font la paire, Paris, Éditions sociales internationales, 1938.

20. Colette Vivier, La Maison des petits bonheurs, Paris, Bourrelier, coll. « Marjolaine », 1940 (Prix Jeunesse 1939), réédité par La Farandole en 1970 et en 1980, puis par Casterman en 1996, avec des illustrations de Serge Bloch.

21. Marie-Cécile Bouju, « Le livre comme arme de propagande », op. cit., p. 56. 
bénéficie de la collaboration de l'Association des écrivains et artistes révolutionnaires (AÉAR) et est finalement édité par les Éditions sociales internationales. Celles-ci reprennent le titre du périodique et développent une collection de livres pour enfants, les « Livrets de Mon Camarade », point de départ d'un véritable secteur éditorial, car les Éditions sociales internationales lancent en parallèle une collection pour la jeunesse, adaptée aux différentes tranches d'âge. Quels sont les textes littéraires et les écrivains publiés dans Mon Camarade? Outre les romans publiés en feuilleton précédemment évoqués, comme Le Mystère du serpent à plumes ${ }^{22}$, la rubrique « Notre page choisie » est intéressante en ce qu'elle révèle quels écrivains et quelles œuvres constituent, selon les rédacteurs en chef du journal, une culture littéraire du jeune communiste, à la fin des années 1930. Les auteurs contemporains proches du Parti communiste, comme Charles Vildrac, Romain Rolland, Henri Barbusse, Jules Romains, André Chamson, Paul VaillantCouturier, y côtoient des écrivains moins révolutionnaires, tels Alphonse Daudet, Erkmann Chatrian, George Sand, Maupassant ou Théophile Gautier. C'est que le propos littéraire de Mon Camarade n'est pas uniquement guidé par un discours de propagande : il s'agit de donner aux jeunes lecteurs une culture littéraire de qualité, où les auteurs classiques comme Homère, Cervantès, Voltaire ou les frères Grimm voisinent avec les écrivains prolétariens (Hermynia zur Mühlen) et le Panthéon de la littérature française (Victor Hugo, Anatole France) et européenne (Selma Lagerlöf, Andersen, Dickens, Tolstoï). Lewis Carroll, écrivain que les surréalistes, en particulier Louis Aragon, ont largement contribué à faire connaître en France ${ }^{23}$, vers 1930, fait aussi une apparition discrète mais significative d'une recherche de nouveauté et de qualité littéraires. Cette qualité est essentielle pour Georges Sadoul qui, en 1938, publie un essai critiquant de façon radicale l'invasion de la presse américaine et italienne en France, supports, notamment par le biais de la bande dessinée, de discours violents, racistes, fascistes, qu'il est impératif, selon lui, de contrer ${ }^{24}$. En développant des pages littéraires dans son journal, Georges Sadoul ne diffuse pas seulement les textes des écrivains communistes : il utilise le périodique comme un creuset littéraire et culturel, tentant de créer une culture littéraire non seulement communiste mais commune aux jeunes lecteurs, donnant à lire de grands textes à de petits enfants, selon l'idée soutenue par des éditeurs importants comme Hetzel dans les années 1860 ou Stock à la fin des années 1920.

22. Il faut ajouter la suite des aventures de Pierrot Lancry, Le Trèfle noir (1936-1937), L'Homme des gouffres (1937-1938), La Mort du loup (1938-1939), mais aussi quelques romans russes (Les Trois Gros, « grand roman humoristique par Oliécha », 1935-1936) et Trotte-Menu chez les chasseurs d'images de René Lelu (1937).

23. Comme 1'a montré Isabelle Nières-Chevrel dans sa thèse Lewis Carroll en France (1870-1985); les ambivalences d'une réception littéraire, Université de Picardie, 1988.

24. Voir Georges Sadoul, Ce que lisent vos enfants, Paris, Éditions sociales internationales, 1938. 
Il est possible ainsi de conclure sur la place importante des éditeurs communistes français dans la diffusion des œuvres pour enfants et sur leur influence en matière de création littéraire auprès des écrivains communistes pour la jeunesse. Marie-Cécile Bouju, dans son Catalogue de la production des maisons d'édition du PCF, 1921-1956 (ENSSIB, 1999), répertorie plusieurs collections pour la jeunesse : la « Bibliothèque des jeunesses communistes », la «Bibliothèque du jeune communiste », déclinée sur le même modèle en Bibliothèque du jeune prolétaire, léniniste, travailleur. Elle montre cependant que les Éditions sociales internationales sont les seules à publier une collection conséquente et durable, avec la collection « Mon Camarade », complétée par les « Livrets de Mon Camarade ». Cette collection est significative du rôle joué par l'idéologie communiste dans les échanges de livres pour la jeunesse dans l'entre-deux-guerres. Selon l'esprit internationaliste des partis communistes européens, la littérature pour la jeunesse a pour vocation de transcender les frontières. Recoupant partiellement la cause pacifiste, l'engagement de la littérature prolétarienne pour la jeunesse correspond ainsi, notamment entre la France et l'Allemagne, à une volonté de donner un souffle nouveau aux échanges franco-allemands, d'élargir l'horizon des jeunes lecteurs et de créer un dynamisme de la création littéraire. En 1938, sur la douzaine de titres proposés par la collection «Mon Camarade », la moitié sont des traductions, deux du russe ${ }^{25}$ et trois de l'allemand. Alors que les traductions de l'anglais dominent dans l'édition pour la jeunesse des années 1930, la collection ne publie qu'une traduction, celle de L'Île au trésor. Évitant de publier des livres issus d'un capitalisme honni, la collection traduit en revanche les Proletarische Märchen ${ }^{26}$ d'Hermynia zur Mühlen, contes prolétariens très engagés, publiés à Berlin en 1924, mais qui sont aussi un livre à l'esthétique inédite, grâce aux magistrales illustrations à l'encre de Chine de Georg Grosz. L'écriture communiste pour la jeunesse ne saurait donc se réduire à la diffusion d'un discours de propagande : traductions, échanges et création se conjuguent pour qu'éditeurs, écrivains et artistes participent au renouveau de la littérature pour la jeunesse de l'entre-deuxguerres, qui trouvera des prolongements au lendemain de la Seconde Guerre mondiale.

Mathilde Lévêque

Université Paris 13 - CENEL

25. L'Épopée du travail moderne : la merveilleuse transformation de l'Union soviétique, par M. Iline et Les Trois méchants gros par Iouri Olécha.

26. Ces contes sont traduits en plusieurs volumes: Ce que disent les amis du Petit Pierre, suivi de La Muraille magique, et Les Trois amis, Le Cheval de fiacre, Le Pont. 